\title{
EFECTO DEL EXTRACTO ETANÓLICO DE SEMILLAS DE Annona muricata (GUANÁBANA) PARA EL CONTROL DE LARVAS DE MOSQUITOS Culex quinquefasciatus
}

\author{
EFFECT OF ETHANOL EXTRACT SEED Annona muricata (SOURSOP) FOR CONTROL OF \\ MOSQUITO LARVAE Culex quinquefasciatus
}

\author{
Gloria Lizeth Parra-Rodríguez, Katherinne Cortes-Daza y Diego Tomas Corradine-Mora ${ }^{\text {cvLAC }}$
}

\begin{abstract}
RESUMEN
Se evaluó el efecto del extracto etanólico de semillas de Annona muricata (guanábana) sobre la susceptibilidad del mosquito Culex quinquefasciatus, en sus cuatro estadios larvarios en condiciones de laboratorio. Se realizaron bioensayos los cuales consistieron en aplicar concentraciones del extracto ( 100 ppm, 250 ppm y 500 ppm), con tres réplicas por cada concentración y un control para cada estadio larvario. Se evidenció 100 \% de letalidad en el Estadio 1 a las 24 horas con las tres concentraciones experimentales evaluadas. En el estadio 2, hubo letalidad del 100 \% con una concentración de 500 ppm a partir de las 12 horas, de igual manera se logró letalidad con las concentraciones de 100 y 250 ppm a partir de las 24 horas. En el estadio 3 la letalidad fue del 96 \% en la concentración de 100 ppm al cabo de 72 horas y del $100 \%$ en las concentraciones de 250 y 500 ppm a las 48 y 12 horas respectivamente. En el estadio 4, hubo letalidad del $100 \%$ a las 24 horas en la concentración de 500 ppm, mientras en las otras dos concentraciones se alcanzaron letalidades del $92 \%$ al final de las 72 horas de monitoreo. Esta investigación permitió confirmar que las semillas de A. muricata (guanábana), tiene un alto porcentaje de efectividad como biopesticida en concentraciones de (100 ppm, 250 ppm y 500 ppm) en cualquier estadio de desarrollo larvario del mosquito C. quinquefasciatus; lo cual permite dar una alternativa natural, viable, económica y amigable con el medio ambiente para el control de esta especie.
\end{abstract}

PALABRAS CLAVE: Annona muricata; Culex quinquefasciatus; biopesticida; extracto etanólico

\begin{abstract}
Under strict laboratory conditions, the effect and susceptibility of using ethanol extract from the seeds of Annona muricata (Soursop) on mosquitoes type Culex quinquefasciatus, in its four larval stages, was evaluated. The following bioassays were performed which consisted of applying concentrations of the extract in (100ppm, $250 \mathrm{ppm}$ and $500 \mathrm{ppm}$ concentrations), with three replicates per concentration and control of each instar. $100 \%$ lethality was demonstrated in Stage 1 at the 24 hour mark, with the three concentrations evaluated. In Stage 2, 100 \% lethality was achieved with a concentration of $500 \mathrm{ppm}$ at the 12 hour mark. The same lethality was achieved with concentrations of 100 and $250 \mathrm{ppm}$ at the 24 hour mark. In stage 3 lethality was $96 \%$ with a concentration of $100 \mathrm{ppm}$ at the 72 hour mark, and $100 \%$ with concentrations of 250 and $500 \mathrm{ppm}$ at 48 and 12 hours respectively. In Stage 4, there was a $100 \%$ lethality at the 24 hour mark with a concentration of $500 \mathrm{ppm}$. The other 2 concentrations resulted in a lethality of $92 \%$ at the 72 hour mark which was the end of the evaluated monitoring time. This study confirmed that the seeds of A. muricata (Soursop), has a high percentage of effectiveness as a bio-pesticide in concentrations of (100 ppm, $250 \mathrm{ppm}$ and $500 \mathrm{ppm}$ ) in any stage of larval development of the C. quinquefasciatus mosquito. This allows for a naturally viable, economical and environmentally friendly alternative for controlling this species.
\end{abstract}

KEY WORDS: Annona muricata; Culex quinquefasciatus; biopesticide; ethanol extract

Dirección de los autores:

Universidad Distrital Francisco José de Caldas, Bogotá, Colombia. Celular: 3002840442; e-mail: lizethparo@gmail.com (G.LP.R). Celular: 3134636188; e-mail: katha_9028@hotmail.com (K.C.D). Celular: 3124870262; e-mail: dtcorradinem@udistrital.edu.co (D.T.C.M). 


\section{INTRODUCCIÓN}

Los mosquitos constituyen un grupo de insectos de gran importancia, debido a que muchas especies producen diversas molestias, además son vectores de agentes causales de enfermedades humanas con gran importancia en salud pública. Uno de estos vectores es el mosquito Culex quinquefasciatus, ya que es el agente transmisor de enfermedades como la filariosis, encefalitis virales equinas, entre otras (Organización Mundial de la Salud, 1992).

Una de las alternativas que más se utiliza desde antes de la segunda guerra mundial para el control y erradicación de dichos vectores es el uso de los insecticidas químicos como los organoclorados, los organofosforados, los carbamatos, entre otros. Su uso sin ningún control natural, su difícil degradación y su larga persistencia en el medio ambiente, han ocasionado efectos significativos en la salud humana como intoxicaciones agudas y crónicas, impactos negativos en los animales y en el ecosistema, contaminando los cuerpos de agua, lo cual constituye un grave peligro para el abastecimiento de agua potable y agua para riego, así mismo en el aire, dejando residuos en suspensión y, por último, causando alteraciones de fertilización en los suelos, disminuyendo la fauna y flora. Como consecuencia de esto, algunos productos agrícolas pierden calidad nutritiva que los hacen no aptos para el consumo humano. Además su uso masivo ha llegado a provocar la resistencia a estos insecticidas por parte de algunas poblaciones del mosquito C. quinquefasciatus (Auditoría General de la República, 2004).

Estudios realizados han demostrado que todas las partes del árbol de Annona muricata (guanábana), poseen propiedades insecticidas, debido a la presencia de diversos compuestos como alcaloides (muricina y muricinina), acetogeninas, entre otros. Estos compuestos bioactivos además de presentar actividad plaguicida, contienen una extensa gama de actividades biológicas como: antitumoral, citotóxica, antimicrobiana, antoprotozoaria y antihelmíntica (García, 2010).

En el estudio "Evaluación larvicida de suspensiones acuosas de Annona muricata Linnaeus (guanábana) sobre Aedes aegypti Linnaeus" se evaluó de forma comparada, la toxicidad de los extractos etanólicos de semillas, flores, hojas, cortezas de A. muricata (guanábana) sobre larvas del IV estadio de A. aegypti.
Se logró 100 \% de efecto tóxico con el extracto de las semillas a concentración de $0,5 \mathrm{mg} / \mathrm{mL}$ al cabo de 24 horas, este mismo resultado se consiguió con las flores a las 48 horas a una concentración mucho mayor, de 10 $\mathrm{mg} / \mathrm{mL}$ y con extracto de hojas a las 36 horas con una concentración de $100 \mathrm{mg} / \mathrm{mL}$ (Bobadilla et al., 2005).

Los resultados de la investigación "Efecto bioinsecticida del extracto etanólico de las semillas Annona cherimolia Miller "chirimoya" y A. muricata Linneaus "guanábana" sobre larvas del IV estadio de Anopheles sp. del año 2002", donde se evaluó la toxicidad de los extractos etanólicos de las semillas de A. cherimolia y A. muricata, mostraron un $100 \%$ de efecto tóxico a las 24 horas, con una concentración de 0,12 mL / $100 \mathrm{~mL}$ de la semilla de A. muricata y 0,8 mL / $100 \mathrm{~mL}$ de la semilla A. cherimolia; evidenciando que la semilla de $A$. muricata tiene un mayor efecto tóxico larvario con una concentración menor (Bobadilla et al., 2002).

Por los motivos expuestos anteriormente y con la perspectiva de incorporar un recurso alternativo para el control de mosquitos $C$. quinquefasciatus, dentro de un manejo sostenible y menos nocivo para nuestro ambiente, se evaluó el efecto del extracto etanólico de semillas de A. muricata (guanábana) sobre larvas de primer, segundo, tercer y cuarto estadio de mosquitos C. quinquefasciatus.

\section{MATERIALES Y MÉTODOS}

Para la evaluación del efecto extracto etanólico de semillas de A. muricata (guanábana) sobre larvas de primer, segundo, tercer y cuarto estadio de mosquitos C. quinquefasciatus, se realizó un estudio cuantitativo que se desarrolló en el Laboratorio de Zoonosis de la Facultad de Medio Ambiente y Recursos Naturales de la Universidad Distrital Francisco José de Caldas; para obtener resultados de la evaluación fueron necesarios realizar siete procedimientos diferentes los cuales se ejecutaron en el siguiente orden:

\section{Obtención del material vegetal}

El material vegetal (semillas de A. muricata) fue obtenido de forma comercial en la ciudad de Bogotá verificando su procedencia de los cultivos del Tolima. Se compraron frutos de los cuales se sacaron dos libras de semillas, las cuales fueron necesarias para luego realizar el extracto. 


\section{Captura y crianza de larvas y adultos de mosquitos $C$. quinquefasciatus}

La captura de los adultos de $C$. quinquefasciatus fue realizada en el Jardín Botánico José Celestino Mutis, estos fueron trasladados a una jaula artesanal, la cual se mantuvo en el Laboratorio de Zoonosis de la Universidad Distrital Francisco José de Caldas, bajo condiciones no controladas de temperatura, luz y humedad relativa. Los organismos ensayo fueron alimentados con una dieta a base de sacarosa. Para la obtención de las larvas fue necesario que las hembras de $C$. quinquefasciatus realizaran hematofagia, para lo cual se introdujo en la jaula de los mosquitos un ratón de laboratorio (Mus musculus), previamente anestesiado.

\section{Observación semanal de las balsas de huevos y larvas}

Desde la primera obtención de balsas con huevos de los organismos de ensayo, se realizaron monitoreos de tres días a la semana y en ocasiones cuatro días a la semana (según la evolución de las balsas con huevos y larvas), las larvas emergieron de las balsas luego de tres o cuatro días de haber sido puestas por las hembras. Las larvas se clasificaron por tamaños y se separaron por estadio para poder realizar los bioensayos. Las larvas tardaron entre dos y tres días en pasar de un estadio al siguiente y, en ocasiones, tardaban hasta cinco días hasta llegar a convertirse en pupas y luego en adultos.

\section{Preparación y determinación de las concentraciones (ppm) del extracto etanólico}

Se preparó el extracto en el Laboratorio de Zoonosis a base de semillas de A. muricata (guanábana), en concentraciones de 100 ppm, 250 ppm y 500 ppm con el siguiente procedimiento:

Posterior al pesaje de la cantidad a utilizar $(2 \mathrm{lb}$ de semillas), las semillas de A. muricata se lavaron con agua limpia y se dejaron secar dispersas sobre una bandeja en un lugar con poca ventilación e iluminación durante siete días, esto con el fin de evitar la contaminación de las semillas por organismos externos. Pasado este tiempo se procedió a triturar las semillas con un molino manual. Luego de la trituración, se licuaron las semillas en tres litros de etanol al $95 \%$ (por cada libra de semillas) durante cinco minutos aproximadamente, este licuado se dejó en reposo durante 72 horas en un recipiente plástico a temperatura ambiente. Posterior al periodo de sedimentación, se realizó la filtración, con el fin de separar los sólidos sedimentables y suspendidos (residuos de las semillas) por medio de filtración al vacío. El extracto filtrado fue almacenado en botellas ámbar plásticas de $500 \mathrm{~mL}$, previamente lavadas y esterilizadas. El extracto crudo obtenido se sometió a evaporación rotatoria (Rotaevaporador IKA RV10) en dos sesiones, con el fin de eliminar el solvente. Todo el material obtenido fue recolectado en frascos de vidrio color ámbar previamente lavados y esterilizados, almacenados a $4{ }^{\circ} \mathrm{C}$, el cual sería la solución madre. De esta solución madre se observaron algunas características organolépticas como color, olor, textura y viscosidad. Se pesaron $0,1 \mathrm{~g}, 0,25 \mathrm{~g}$ y $0,50 \mathrm{~g}$ de la solución madre de semillas de A. muricata (guanábana), que equivalen a las concentraciones que se utilizaron en los bioensayos.

\section{Realización de los bioensayos en los cuatro estadios larvarios del mosquito $C$. quinquefasciatus}

Los bioensayos se llevaron a cabo en el Laboratorio de Zoonosis, donde se utilizaron 48 recipientes plásticos de 21 de capacidad, a los cuales se les adicionó 11 de agua, la concentración correspondiente $(100,250$ y 500 ppm) del extracto crudo etanólico diluido con $3 \mathrm{ml}$ de etanol y $1 \mathrm{~g}$ de alimento concentrado para conejos (con la finalidad de proporcionar nutrientes a las larvas. Los controles se formularon con $1 \mathrm{l}$ de agua y $1 \mathrm{~g}$ de alimento (todo fue medido con la ayuda de un balón aforado previamente lavado y esterilizado de $1000 \mathrm{~mL}$ ). Como control de viabilidad, se realizaron cuatro bioensayos con $3 \mathrm{ml}$ de etanol, $1 \mathrm{l}$ de agua, $1 \mathrm{~g}$ de alimento y 50 larvas por cada estadio, con el fin de evidenciar que el etanol no causara letalidad en las larvas.

En total se realizaron 12 bioensayos, distribuidos en tres tratamientos por cada estadio de desarrollo larval (100, 250 y $500 \mathrm{ppm})$, sometidos a tres replicas por cada concentración con su respectivo control, utilizando 50 larvas por réplica. Por cada estadio, se utilizaron 600 larvas repartidas en los tres tratamientos experimentales y un control, para un total de 2400 larvas de $C$. quinquefasciatus. 


\section{Evaluación de la letalidad}

Las lecturas de letalidad se realizaron a las $12,24,36$, 48, 60 y 72 horas, luego de haber aplicado el extracto crudo etanólico, donde las larvas se consideraron muertas cuando no reaccionaron al momento de ser tocadas con un puntero metálico de punta roma en la región cervical.

\section{Análisis estadísticos de esta evaluación}

Se determinaron medidas de tendencia central, análisis de varianza (ANOVA) factorial con prueba pos hoc de Tukey, con la finalidad de demostrar la existencia de diferencias significativas entre los promedios de mortalidad de cada una de las concentraciones definidas y entre cada uno de los estadios.

\section{RESULTADOS}

Una vez realizados los bioensayos necesarios para evaluar la toxicidad larvaria del extracto etanólico de semillas de A. muricata (guanábana), se procedió a organizar y analizar cada una de las variables contenidas en este estudio. Se separaron los resultados por estadios y por concentraciones, con el fin de conocer más detalladamente cómo fue el comportamiento de las larvas del mosquito $C$. quinquefasciatus frente al extracto etanólico de las semillas, cuál fue el porcentaje de mortalidad en los diferentes estadios larvarios y en qué tiempos de exposición se dieron estos porcentajes.

Los resultados obtenidos en los controles en larvas de primero, segundo, tercero y cuarto estadio de mosquitos C. quinquefasciatus, se hicieron en las mismas condiciones, fechas y horas en las que fueron realizados los bioensayos, con la diferencia que a los controles no se les adicionó extracto etanólico de semillas de A. muricata (Guanábana), ni etanol; esto con el fin de comprobar que en los bioensayos la mortalidad de las larvas fuera causada por la adición de extracto y no por algún otro factor, como, que el agua o comida estuvieran contaminadas, que los utensilios de medición y los recipientes no estuvieran bien lavados o que durante el periodo de experimentación cayeran a los bioensayos algunas partículas del ambiente que causaran la mortalidad de las larvas.

\section{Estadio 1}

En larvas de estadio 1, se evidenció a las 24 horas de haber aplicado el extracto de semillas de A. muricata, una letalidad del $100 \%$ con las tres concentraciones evaluadas, aunque a las 12 horas en la concentración más alta (500 ppm), ya se evidenciaba un $98 \%$ de mortalidad, en el mismo tiempo, la concentración de (100 ppm y 250 ppm) alcanzaron una letalidad del $90 \%$ (Tabla 1).

Tabla 1. Valores porcentuales de mortalidad dependiendo el estadio de Culex quinquefasciatus, la concentración del extracto etanólico de semillas de Annona muricata y el monitoreo.

$\begin{array}{llll}\text { CONCENTRACIÓN (ppm) } & 100 & 250 & 500\end{array}$

\begin{tabular}{ccccccccccccc}
\hline ESTADIO & & & & & & & & & & & & \\
\cline { 1 - 7 } MONITOREO (Horas) & & $\mathbf{2}$ & $\mathbf{3}$ & $\mathbf{4}$ & $\mathbf{1}$ & $\mathbf{2}$ & $\mathbf{3}$ & $\mathbf{4}$ & $\mathbf{1}$ & $\mathbf{2}$ & $\mathbf{3}$ & $\mathbf{4}$ \\
\hline 12 & 90 & 92 & 22 & 8 & 90 & 98 & 86 & 84 & 98 & 100 & 100 & 100 \\
\hline 24 & 100 & 100 & 88 & 82 & 100 & 100 & 92 & 90 & 100 & 100 & 100 & 100 \\
\hline 36 & 100 & 100 & 90 & 86 & 100 & 100 & 96 & 90 & 100 & 100 & 100 & 100 \\
\hline 48 & 100 & 100 & 94 & 88 & 100 & 100 & 100 & 92 & 100 & 100 & 100 & 100 \\
\hline 60 & 100 & 100 & 94 & 90 & 100 & 100 & 100 & 92 & 100 & 100 & 100 & 100 \\
\hline 72 & 100 & 100 & 96 & 92 & 100 & 100 & 100 & 92 & 100 & 100 & 100 & 100 \\
\hline
\end{tabular}


Los resultados que se exponen en la figura 1 demuestran que el primer estadio larvario de mosquitos C. quinquefasciatus es bastante sensible al extracto de semillas de A. muricata. Los porcentajes de mortalidad en el control indican que los factores externos no influyeron en el momento de evaluar la letalidad de las diferentes concentraciones del extracto.

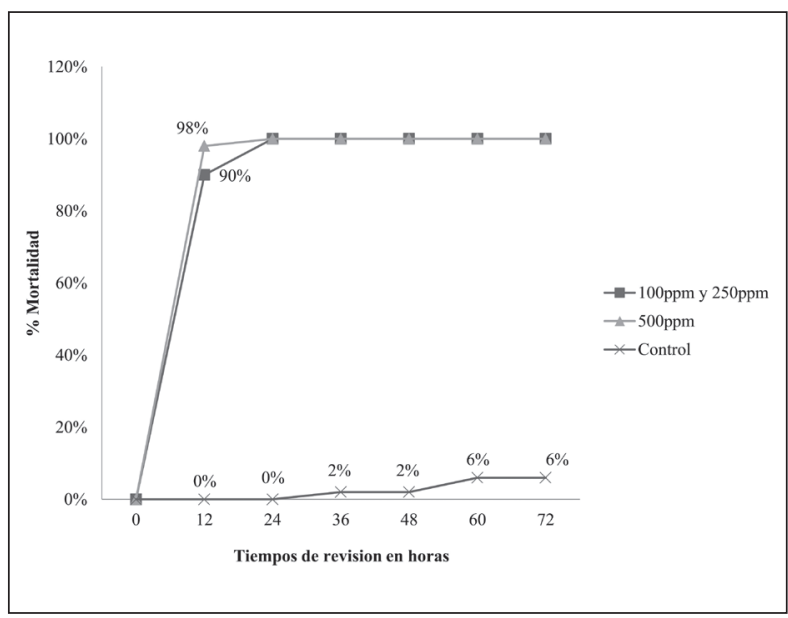

Figura 1. Mortalidad de larvas en primer estadio de mosquitos Culex quinquefasciatus con las tres concentraciones del extracto etanólico de semillas de Annona muricata (guanábana) y su respectivo control.

\section{Estadio 2}

En larvas de estadio 2, arrojaron resultados un $100 \%$ de mortalidad de las larvas, en el menor tiempo de exposición a las 12 horas en la concentración más alta (500 ppm), aunque en las otras dos concentraciones (100 ppm y 250 ppm) y en el mismo lapso de tiempo, se evidenciaron mayores porcentajes de mortalidad 92 \% y del $98 \%$ respectivamente (Tabla 1).

Los resultados que se exponen en la figura 2, muestran que el segundo estadio larvario de mosquitos es igualmente sensible a las larvas de estadio 1 al extracto de semillas de A. muricata. Los porcentajes de mortalidad en el control pudieron depender de las variaciones de temperatura, luz, y humedad relativa, disponibilidad de alimento y del comportamiento normal del ciclo de vida de las larvas.

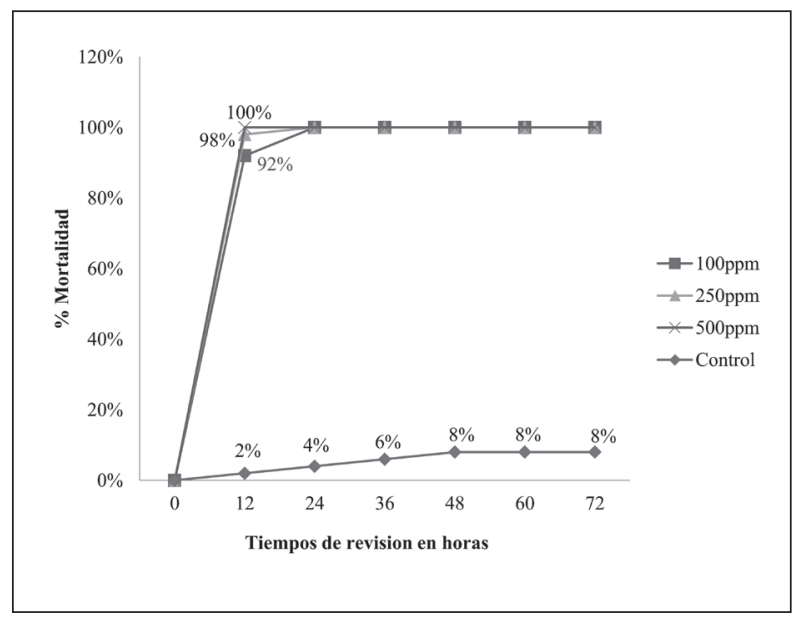

Figura 2. Mortalidad de larvas en segundo estadio de mosquitos Culex quinquefasciatus con las tres concentraciones del extracto etanólico de semillas de Annona muricata (guanábana) y su respectivo control.

\section{Estadio 3}

En la figura 3 se muestra como la mortalidad del $100 \%$ en larvas de estadio 3 , se evidenció a las 48 horas de haber aplicado el extracto de semillas de A. muricata en la concentración de (250 ppm), sin embargo, a las 12 horas en la concentración más alta (500ppm) también se evidenció este porcentaje de mortalidad y en la concentración de (100ppm), se encontró un porcentaje del $96 \%$ de letalidad a las 72 horas (Tabla 1).

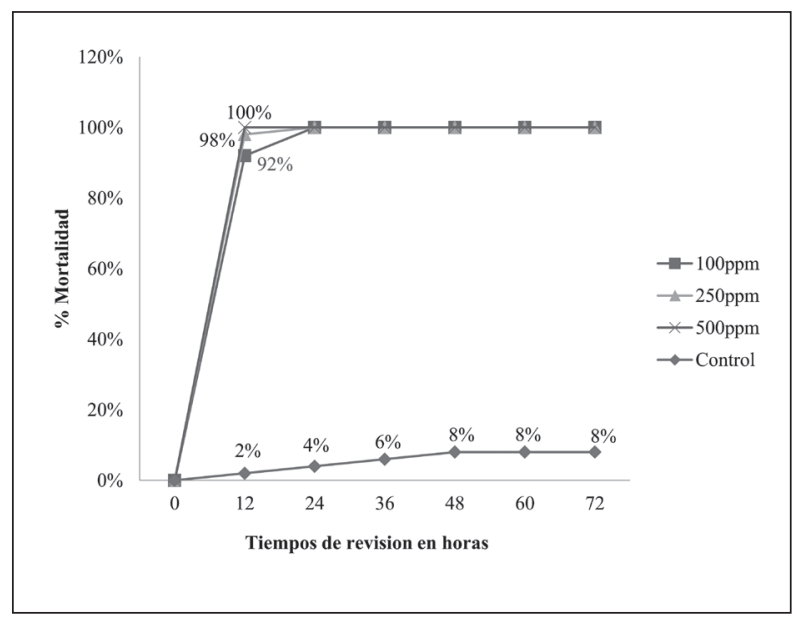

Figura 3. Mortalidad de larvas en tercer estadio de mosquitos Culex quinquefasciatus con las tres concentraciones del extracto etanólico de semillas de Annona muricata (guanábana) y su respectivo control. 
Los porcentajes de letalidad en el control de este estadio fueron mínimos en comparación de los obtenidos en el estadio 1 y 2 , posiblemente a que el estadio 3 puede presentar mayor resistencia a los factores externos por su desarrollo alcanzado.

\section{Estadio 4}

En la figura 4 se observa que en larvas de estadio 4 arrojaron resultados donde se evidenció que a las 12 horas de haber aplicado el extracto de semillas de A. muricata en la concentración más alta $(500$ ppm) se alcanzó el mayor efecto tóxico con un 100 \% de mortalidad, aunque en la concentración media (250 ppm) en el mismo lapso de tiempo se evidenció un 84 $\%$ de mortalidad y con la concentración mínima (100 ppm), alcanzó una mortalidad del $92 \%$ al cabo de las 72 horas (Tabla 1).

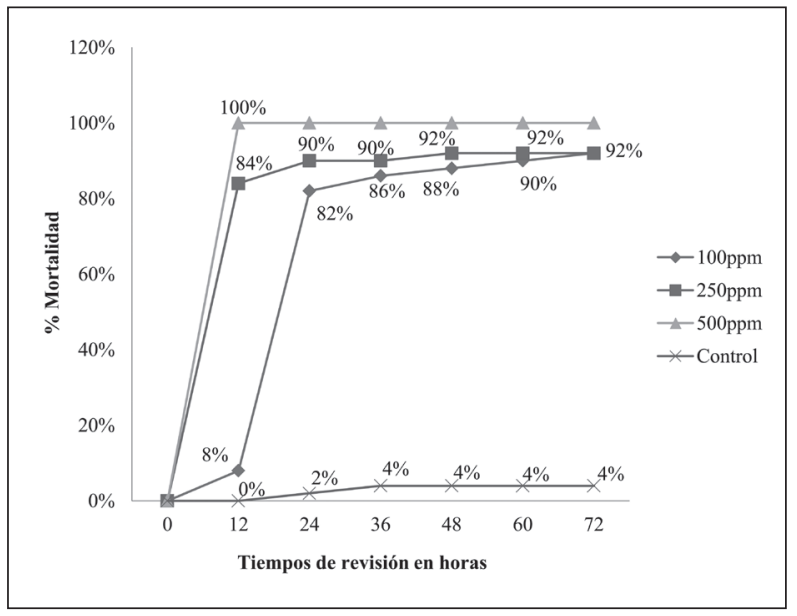

Figura 4. Mortalidad de larvas en cuarto estadio de mosquitos Culex quinquefasciatus con las tres concentraciones del extracto etanólico de semillas de Annona muricata (guanábana) y su respectivo control.

Respecto a los controles, se puede apreciar que existe un porcentaje mínimo de mortalidad de las larvas en los cuatro estadios, lo cual se debe al ciclo de vida normal del mosquito C. quinquefasciatus, ya que en el proceso larvario, al pasar de un estadio al siguiente existen larvas que no lo logran, las cuales hacen un porcentaje mínimo, esto lo sustenta un estudio realizado por estudiantes de la facultad de medicina de la Universidad Nacional de Colombia al ciclo de vida del mosquito C. quinquefasciatus (Salazar y Moncada, 2004).

\section{DISCUSIÓN}

Se han destacado algunas plantas por su efecto nocivo sobre larvas de mosquitos mediante extractos orgánicos y no orgánicos, entre esas se encuentra las del género Annona, tales como A. bullata, A. glabra, A. muricata y A. squamosa (Bobadilla et al., 2002).

Con la realización de los bioensayos se halló que el extracto etanólico de semillas de A. muricata presenta una notoria actividad biológica en los cuatro estadios de las larvas del mosquito C. quinquefasciatus. En estos resultados se puede evidenciar que las larvas del estadio 1 y 2 tuvo una sensibilidad del $100 \%$ a los metabolitos presentes en el extracto etanólico en las tres concentraciones letales con un tiempo de 24 horas; de forma contraria en los estadios 3 y 4 alcanza a tener un poco de resistencia en la concentración de (100 ppm) con una letalidad a las 72 horas, con la concentración de ( 250 ppm) al cabo de 48 horas y con la concentración alta de (500 ppm) con un tiempo mínimo de 12 horas. Con el análisis de varianza (ANOVA) factorial con prueba pos hoc de Tukey, la mortalidad de las larvas tanto en la concentración más baja como en la más alta es la misma, ya que no hay una diferencia notable entre promedios debido a que las tres concentraciones (100 ppm, 250 ppm y 500 ppm) afectaron de igual manera los cuatro estadios de las larvas, aunque se hubiera pensado que si tuvieran variación los promedios de las larvas de tercer y cuarto estadio.

En el informe "Evaluación larvicida de suspensiones acuosas de Annona muricata Linnaeus (guanábana) sobre Aedes aegypti Linnaeus (Díptera, Culicidae) del año 2005" sobre larvas del IV estadio de Aedes aegypti, el mayor efecto tóxico correspondió a la suspensión de las semillas con un $100 \%$ de mortalidad a las 24 horas a 0,5 mg / mL (Bobadilla et al., 2005); mientras que en otro estudio "Actividad insecticida de extractos vegetales sobre Aedes aegypti (Diptera: Culicidae) vector del dengue en Colombia del año 2007" se evaluó la toxicidad de diferentes concentraciones de los extractos etanólicos obtenidos de las semillas de Annona muricata, y de los frutos maduros de Melia azedarach, y Ricinus communis sobre larvas de cuarto estadio de Aedes aegypti, los extractos evaluados produjeron mortalidad en las larvas de Aedes Aegypti a las 24 horas, se observó que el extracto de Ricinus communis es el más activo con una concentración letal media de 860 ppm, seguido por el extracto de Annona muricata con una concentración letal de 50 \% en 900 ppm (Parra et al., 2007). 
Evaluando los dos estudios anteriores y el estudio presente con la misma concentración del extracto pero con diferente género de mosquito, en el primer estudio las larvas en su cuarto estadio de Aedes Aegypti Linnaeus tuvo un porcentaje de letalidad del $100 \%$ al cabo de 24 horas, y en esta investigación tuvo un porcentaje alto de letalidad del $100 \%$ al cabo de 12 horas, en el segundo estudio se usó una concentración mayor de extracto con diferente mosquito y tuvo un porcentaje de letalidad del $50 \%$ al cabo de 24 horas, de aquí se puede evidenciar que el extracto de semilla de A. muricata es más efectivo en el género de $\mathrm{C}$. quinquefasciatus que en la A. aegypti. Aunque son de la misma familia tienen ciertas diferencias que se pueden evidenciar en la efectividad que tiene este extracto para su control plaguicida.

Debido a que la semilla A. muricata contiene anonacina, acimicina y bulatacina ingredientes activos insecticidas contra larvas de mosquitos (Rodríguez, 2000), en el estudio "Toxicidad de aceites, esencias y extractos vegetales en larvas de mosquito Culex quinquefasciatus Say (Diptera: Culicidae)" se usó también la flor y hoja pero se evidencia que la mortalidad obtenida al 5 y 15 $\%$ no fue mayor al 15 \% y que la mejor opción al usar la A. muricata, es usar su semilla ya que la mortalidad fue del 15,6 \% en larvas de cuarto estadio de C. quinquefasciatus (Pérez et al., 2004).

El uso de extractos vegetales como insecticidas y larvicidas, tiene la ventaja de que los niveles de toxicidad son muy elevados, con relación a la acción de compuestos individuales químicamente puros, a la vez que tienen menos riesgo de generar resistencia por la complejidad de su composición. En los últimos tiempos se están realizando evaluaciones de insecticidas naturales a partir de extractos de diferentes partes de la biomasa de una planta, con el propósito de producir efecto sinérgico sobre algún organismo blanco (McLaughlin et al., 1998) y obtener una mayor toxicidad.

Los resultados obtenidos en esta investigación son promisorios para el control de larvas de $C$. quinquefasciatus y posiblemente para el control de larvas de otras especies de mosquitos de la familia Culicidae. Sin embargo, hay que tener en cuenta que la respuesta de cada especie de mosquito puede ser variable con respecto a las dosis diagnósticas halladas para $C$. quinquefasciatus.

\section{CONCLUSIONES}

Se comprobó que las semillas de A. muricata tienen un alto potencial tóxico contra las larvas del mosquito $C$. quinquefasciatus en sus cuatro estadios larvarios.

Con la concentración mínima utilizada en esta investigación de 100 ppm y a partir de 12 horas de exposición ya se evidencia la actividad biopesticida del extracto etanólico de semillas de A. muricata sobre larvas de $C$. quinquefasciatus.

Con una concentración mínima (100 ppm) se obtuvo una letalidad del $100 \%$ en los estadios 1 y 2 al cabo de las 24 horas, en el estadio 3 alcanzó una toxicidad del $96 \%$ y en el estadio 4 con un $92 \%$ a las 72 horas.

Con una concentración de (250 ppm) se obtuvo una mortalidad del $100 \%$ en los estadios 1 y 2 a un tiempo de 24 horas y, en el estadio 3 al cabo de las 48 horas y en el 4 estadio solo alcanzó una letalidad máxima de $92 \%$ al cabo de las 72 horas.

Con la concentración máxima (500ppm) se alcanzó una letalidad del $100 \%$ en todos los estadios a partir de las 12 horas.

El estadio 1 de las larvas del mosquito $C$. quinquefasciatus presentó mayor afección por el extracto etanólico de $A$. muricata, comparado con el estadio 4, el cual presentó mayor resistencia al extracto evaluado.

Al realizar los bioensayos de los controles indicaron que los factores externos no influyeron en el momento de evaluar la letalidad de las diferentes concentraciones del extracto biopesticida.

\section{BIBLIOGRAFÍA}

Auditoría General de la República. 2004. Uso y manejo de plaguicidas en Colombia. Auditoria analítica de gestión._ URL:_http://www.mamacoca.org/docs_de_base/Fumigas/ Auditoria_Gnl_colombia_plaguicidas_marzo2004.pdf. Consultado: 22 de Julio de 2011.

Bobadilla, A., Zavaleta, E., Gil, F., Pollack, V. y Sisniegas, G. 2002. Efecto bioinsecticida del extracto etanólico de las semillas de Annona cherimolia Miller "chirimoya" y A. muricata Linnaeus "guanábana” sobre larvas del IV estadio de Anopheles sp. Revista Peruana de Biología 9(2): 64-73. 
Bobadilla, M., Zavaleta, G., Zavala, F., Mostacero, J. y Taramona, L. 2005. Evaluación larvicida de suspensiones acuosas de Annona muricata Linnaeus "guanábana” sobre Aedes aegypti Linnaeus (Diptera, Culicidae). Revista Peruana de Biología 12(1): 145-152.

García, M. 2010. La guanábana (Annona muricata L.). Propiedades y usos. Revista CitriFrut 27(1): 69-70.

McLaughlin, J., Lingling, R. y Jon, A. 1998. The use of biological assays to evaluate botanicals. Drug Information Journal 32: 513-524.

Organización Mundial de la Salud (OMS). 1992. Resistencia de los vectores de enfermedades a los plaguicidas, $15^{\circ}$ informe del comité de expertos de la OMS en biología de los vectores y lucha antivectorial. Ginebra.
Parra, G.J., García, C.M. y Cotes, J.M. 2007. Actividad insecticida de extractos vegetales sobre Aedes aegypti (Diptera: Culicidae), vector del dengue en Colombia. Revista CES Medicina 21(1): 47-54.

Pérez, P., Cesáreo, R., Lara, R., Montes, B. y Ramírez, V. 2004. Toxicidad de aceites, esencias y extractos vegetales en larvas de mosquito Culex quinquefasciatus Say (Diptera: Culicidae). Acta Zoológica Mexicana 20(1): 141-152.

Rodríguez, H. 2000. Plantas contra plagas: potencial practico de ajo, anona, nim, chile y tabaco. Red de acción sobre plaguicidas y alternativa en México, Texcoco, Estado de México.

Salazar, M. y Moncada, L. 2004. Ciclo de vida de Culex quinquefasciatus Say, 1826 (Diptera: Culicidae) bajo condiciones no controladas en Bogotá. Biomédica 24(4): 385-392.

Fecha de recepción: 25/05/2016

Fecha de aceptación: 03/11/2016

Publicado en línea: 07/04/2017

Para citar este artículo: Parra-Rodríguez, G.L., Cortes-Daza, K. y Corradine-Mora, D.T. 2017. Efecto del extracto etanólico de semillas de Annona muricata (guanábana) para el control de larvas de mosquitos Culex quinquefasciatus. Revista Intropica Vol. 12(1): 23 - 30.

DOI: http://dx.doi.org/10.21676/23897864.2034 